\title{
Ponto de Corte do Índice Homeostatic Model Assessment for Insulin Resistance (HOMA-IR) Avaliado pela Curva Receiver Operating Characteristic (ROC) na Detecção de Síndrome Metabólica em Crianças Pré-Púberes com Excesso de Peso
}

\section{artigo original}

ISABEL ReY MADEIRA

Cecilia N. Miranda Carvalho

FERNANDA MUSSI Gazolla

HAROLDO José de Matos

Marcos Antonio Borges

Maria Alice NeVes Bordallo

Departamento de Pediatria da

Faculdade de Ciências Médicas

(FCM) da Universidade do

Estado do Rio de Janeiro (UERJ)

(IRM); Departamento de

Nutrição Aplicada do Instituto de Nutrição da UERJ (CNMC);

Unidade Docente Assistencial

(UDA) de Endocrinologia do

Hospital Universitário Pedro

Ernesto (HUPE), UERJ (FMG);

Departamento de Tecnologias da Informação e Educação em Saúde da FCM-UERJ (HJM); UDA de Endocrinologia do HUPE da UERJ (MAB); UDA de

Endocrinologia do

Departamento de Medicina

Interna da FCM-UERJ (MANB);

Rio de Janeiro, RJ, Brasil.

Recebido em 11/8/2008 Aceito em 6/10/2008

\section{RESUMO}

Objetivo: Estabelecer, entre os pontos de corte do índice homeostatic model assessment for insulin resistance (HOMA-IR), citados na literatura, o melhor em identificar a síndrome metabólica (SM) em crianças com obesidade e sobrepeso. Métodos: Foram estudadas 140 crianças pré-púberes. A definição de SM foi adaptada da International Diabetes Federation. Para cada ponto de corte de HOMA-IR foram estimados sensibilidade e especificidade, tomandose como desfecho a SM. Uma curva receiver operating characteristic (ROC) foi construída com estes valores. Resultados: $O$ grupo estudado constituiu-se de 106 crianças com obesidade ( 37 meninas e 69 meninos) e 34 com sobrepeso (19 meninas e 15 meninos), média de idade $6,5 \pm 2,3$ anos. A acurácia da curva ROC foi $72 \%$, e o melhor ponto de corte foi 2,5 , com sensibilidade $61 \%$ e especificidade $74 \%$. Conclusões: $\mathrm{O}$ índice HOMA-IR pode ser útil para detectar a SM, e o ponto de corte 2,5 mostrou-se o melhor para crianças pré-púberes com obesidade e sobrepeso. (Arq Bras Endocrinol Metab 2008; 52/9:1466-1473)

Descritores: Criança; Curva ROC; Obesidade; Resistência à insulina; Síndrome metabólica

\section{ABSTRACT}

Cut-off Point for Homeostatic Model Assessment for Insulin Resistance (HOMA-IR) Index Established from Receiver Operating Characteristic (ROC) Curve in the Detection of Metabolic Syndrome in Overweight Pre-Pubertal Children.

Objective: To establish, among the cut-off values for the homeostatic model assessment for insulin resistance index (HOMA-IR) cited in the literature, the best in identifying metabolic syndrome (MS) in obese and overweight children. Methods: A total of 106 pre-pubertal children were studied. The definition of MS was adapted from that of the International Diabetes Federation. For each cut-off values for HOMA-IR, it was estimated sensibility and specificity for MS. A receiver operating characteristic (ROC) curve was generated using these values. Results: The study included 106 obese $(37$ girls and 69 boys) and 34 overweight (19 girls and 15 boys) children aged 6,5 $\pm 2,3$ years. The accuracy of the ROC curve was $72 \%$, and the best cut-off value for HOMAIR was 2,5 , with sensitivity of $61 \%$ and specificity of $74 \%$. Conclusions: HOMA-IR may be useful to detect MS and the cut-off 2,5 seems to be the best in obese and overweight pre-pubertal children. (Arq Bras Endocrinol Metab 2008; 52/9:1466-1473)

Keywords: Children; ROC curve, Obesity; Insulin resistance; Metabolic syndrome 


\section{INTRODUÇÃO}

obesidade, inclusive em crianças, é bastante prevalente na sociedade atual, não só nos países desenvolvidos (1), mas, também, em países em desenvolvimento, como o Brasil (2). A doença cardiovascular aterosclerótica, sua complicação mais importante, constitui a maior causa de morte em adultos no mundo ocidental (3), e já está presente em obesos desde a infância, conforme demonstra importantes estudos epidemiológicos, como o de Bogalusa (4). Os principais fatores de risco para a doença cardiovascular são, além da obesidade, a hipertensão arterial, a dislipidemia e o diabetes melito tipo 2 (DM2), reunidos na denominada síndrome metabólica. Nesta síndrome, a resistência insulínica e a hiperinsulinemia explicariam o papel central da primeira e sua relação com as outras anormalidades (5). A atuação destes fatores parece se iniciar na infância (6-12).

A resistência insulínica é definida como a ineficiência da insulina plasmática, sob concentrações usuais, em promover adequada captação periférica de glicose, suprimir a gliconeogênese hepática e inibir a produção de lipoproteína de muito baixa densidade. Suspeita-se de resistência insulínica em face de evidências clínicas fortes, como aquelas que compõem a síndrome metabólica. A confirmação se faz com medidas de insulina e glicose plasmáticas, por meio de índices com base na insulinemia e na glicemia de jejum, ou após teste oral de tolerância à glicose (TOTG), ou por intermédio do modelo mínimo fundamentado em coletas de amostras após glicose intravenosa ou, ainda, a partir dos estudos de clampe de insulina e glicose (11). Estes últimos são os padrões-ouro, mas são procedimentos invasivos e de difícil execução em pediatria. Nenhum destes métodos tem ponto de corte bem estabelecido para a faixa etária pediátrica.

Um desses índices, o homeostasis model assessment for insulin resistance (HOMA-IR), é calculado multiplicando-se a glicemia $(\mathrm{mmol} / \mathrm{L})$ pela insulina $(\mu \mathrm{UI} / \mathrm{mL})$, ambas em jejum, e dividindo-se por 22,5 (12). O índice é amplamente utilizado em adultos, nos quais o ponto de corte mais aceito, acima do qual se define a resistência insulínica, é 2,5 (12). No entanto, este ponto de corte não é consensual, já que vários estudos propõem outros valores, conforme recente revisão sobre o tema (13).

O índice HOMA-IR tem sido validado em crianças e adolescentes por meio da comparação com índices fundamentados em teste oral de tolerância à glicose (14-18) e com clampe de insulina e glicose $(19,20)$. A partir destas tentativas de validação, alguns autores procuram estabelecer pontos de corte de HOMA-IR para resistência insulínica para esta faixa etária. Barja e cols. (21) o definiram como três desvios-padrão positivos da distribuição de um grupo de eutróficos, encontrando o valor de 3,8. Manios e cols. (22) utilizaram o percentil 97 de um grupo de eutróficos e obesos, encontrando como ponto de corte o valor de 2,1, enquanto Cuartero e cols. (23) utilizaram o percentil 90 de indivíduos eutróficos, encontrando o valor de 3,43. Pela construção de curvas receiver operating characteristic (ROC), em estudos em indivíduos com obesidade, Keskin e cols. (17) chegaram ao valor de 3,16, e Atabek e Pirgon (18) ao valor 2,7 , enquanto Tresaco e cols. (24), em outro estudo, avaliando eutróficos e obesos, concluíram que valores em torno de três são adequados. Todos estes estudos incluíram crianças púberes. Já o estudo multicêntrico italiano de d'Annunzio e cols. (25) forneceu o percentil 95 de HOMA-IR para pré-púberes de 2,5.

$\mathrm{Na}$ faixa etária pediátrica, a obesidade parece ser importante gatilho de resistência insulínica (11), o que torna as crianças obesas um grupo de risco, levando os pesquisadores a buscarem indicadores clínicos e laboratoriais neste grupo. A despeito disso, não existe consenso para a definição da síndrome metabólica em crianças, visto que recente revisão sobre o tema encontrou 40 diferentes definições adaptadas a partir daquelas propostas para adultos. Algumas das definições propostas, inclusive, incluem o HOMA-IR como um dos componentes (26).

Recentemente, a International Diabetes Federation (IDF) propôs, para crianças maiores de 10 anos, definição de síndrome metabólica com base na presença de circunferência da cintura aumentada mais pelo menos dois dos elementos que se seguem: hipertrigliceridemia, HDL-colesterol baixo, hipertensão arterial e glicemia de jejum alterada ou DM2 (27).

Diante do panorama descrito, o objetivo deste trabalho foi determinar, por intermédio dos pontos de corte do índice HOMA-IR descritos na literatura, aquele que mais bem identifique a síndrome metabólica em crianças pré-púberes obesas e com sobrepeso.

\section{MATERIAIS E MÉTODOS}

Trata-se de estudo de observação, transversal, com crianças oriundas do Ambulatório de Pediatria Geral do Hospital Universitário Pedro Ernesto da Universidade do Estado do Rio de Janeiro (HUPE-UERJ). Foram 
convidadas a participar da pesquisa todas as crianças impúberes, segundo avaliação clínica para o critério de Tanner (28), com idades de 2 a 11 anos, com obesidade ou sobrepeso, saudáveis sobre outros aspectos, que não estivessem participando em algum programa para perda de peso, e que o responsável legal concordasse com a participação na pesquisa, após ser informado e esclarecido.

O estudo incluiu todas as crianças elegíveis para a pesquisa que tiveram consentimento livre e esclarecido assinado pelo responsável legal. Fazendo-se um cálculo de tamanho amostral para o inquérito, considerando a população de crianças atendidas no ambulatório de Pediatria Geral do HUPE-UERJ, estimando-se proporção esperada de $10 \%$ para obesidade (29), encontrou-se tamanho amostral de 137 crianças para nível de significância de 5\% (30).

As crianças foram submetidas à avaliação clínica completa, segundo o protocolo. A pesagem foi sem sapatos e com roupas leves, em balança da marca Filizola, com resolução do peso de $100 \mathrm{~g}$, e suas estaturas foram medidas sem sapatos, em estadiômetro de parede do tipo Halpender-Holtain da marca Tonelli, com resolução de $1 \mathrm{~mm}$. A medida da circunferência da cintura foi tomada com a criança despida, à altura logo acima da borda lateral mais alta do ilíaco direito, ao final de uma expiração normal, segundo as recomendações do Third National Health and Nutrition Examination Survey (NHANES III) (31), com fita antropométrica milimetrada, da marca Mabbis, modelo Gulik.

A medida da pressão arterial foi tomada no braço direito, com a criança sentada, em repouso, com esfigmomanômetro da marca Tycos, usando-se manguitos de tamanhos adequados. O método empregado foi o auscultatório, com as pressões sistólica e diastólica correspondendo às fases I e V de Korotkoff, seguindo as recomendações de Hoekelman (32).

A coleta de sangue para os exames laboratoriais foi feita após jejum de 12 horas. Para a dosagem de glicose, colesterol total, HDL-colesterol e triglicérides, foi empregado o equipamento Konelab, com o kit BT 3000 Winer, que utiliza: para glicose, o método enzimático GOD-PAP (oxidase); para colesterol, o método enzimático CHOP-POD (estearase-oxidase); para triglicérides, o método enzimático GPO/PAP (oxidase); e para HDL-colesterol, o método colorimétrico sem precipitação (enzimático colorimétrico) (Winterlab, Rosário, Santa Fé, Argentina). A dosagem de insulina foi realizada no equipamento GAMA-Cl2, por meio do método Coat- $A$-Count, por radioimunoensaio fase sólida marcado com iodo 125 (DPC, Los Angeles, CA, Estados Unidos).

Para definição de obesidade e sobrepeso, utilizaramse os padrões de índice de massa corporal (IMC) propostos pelo Centers for Diseases Control and Prevention americano de 2000. O critério estabelecido para obesidade é o de IMC maior ou igual ao percentil 95 para o sexo e a idade, e para sobrepeso o de IMC maior ou igual ao percentil 85, mas menor que o 95 (33).

A classificação empregada para síndrome metabólica foi adaptada do IDF para crianças acima de 10 anos - circunferência da cintura aumentada, e, pelo menos, mais dois dos seguintes critérios: glicemia de jejum alterada ou DM2, HDL-colesterol baixo, triglicérides altos e hipertensão arterial (27).

A circunferência da cintura foi definida como aumentada quando maior ou igual ao percentil 90 para sexo e idade do NHANES III, na tabela que combina as etnias americano-européia, americano-africana e americano-mexicana (31).

A hipertensão arterial foi definida segundo os critérios do National High Blood Pressure Education Program Working Group on High Blood Pressure in Children and Adolescents dos Estados Unidos (34), que são os recomendados nacionalmente na I Diretriz de Prevenção da Aterosclerose na Infância e na Adolescência (35).

Os pontos de corte adotados para glicemia, HDLcolesterol e triglicérides, todos em jejum, foram os recomendados na I Diretriz de Prevenção da Aterosclerose na Infância e na Adolescência: glicemia de jejum alterada: valores maiores ou iguais a $100 \mathrm{mg} / \mathrm{dL}$; HDLcolesterol baixo: valores menores que $45 \mathrm{mg} / \mathrm{dL}$; triglicérides aumentados: valores maiores ou iguais a $130 \mathrm{mg} / \mathrm{dL}(35)$.

Os dados coletados foram arquivados em planilhas do Excel, versão 7 (MapInfo Corporation, Troy, NY, Estados Unidos). Para a análise das tabelas usadas para os cálculos de sensibilidade, especificidade e para a geração da curva ROC foi utilizado o programa Epi Info, versão 6.0 (CDC, Atlanta, GA, Estados Unidos). Para os cálculos estatísticos, com nível de confiança de 95\%, e para a construção da curva ROC, foi empregado o programa Epi Data, versão 3.1 (The Epi Data Association, Ondense, Denmark).

Para estimar a sensibilidade e a especificidade do índice HOMA-IR para o desfecho síndrome metabólica, 
foram eleitos os seguintes pontos de corte, fundamentados nos estudos com crianças e adolescentes encontrados na literatura: $2 ; 2,5 ; 3,16 ; 3,45$ e 3,8 (17,18,21-25). Com estes valores, foi construída a curva ROC.

A curva ROC é uma curva construída por meio da representação gráfica da sensibilidade (taxa de verdadeiro-positivo) versus l-especificidade (taxa de falso-positivo) de um teste diagnóstico, ao longo da faixa de possíveis valores de ponto de corte, em que o melhor é aquele que fica no ombro da curva. O melhor ponto de corte também é aquele que tem o maior índice de Youden, cujo valor é calculado por meio da fórmula: sensibilidade + especificidade - 1. Este índice permite avaliação da sensibilidade e da especificidade, assumindo-se importância igual para as duas. A área sob a curva ROC determina a acurácia do teste diagnóstico e, quanto maior a área, melhor o teste (36).

Este estudo foi aprovado pelo Comitê de Ética e Pesquisa do Hospital Universitário Pedro Ernesto, da Universidade do Estado do Rio de Janeiro, onde está cadastrado com o n ${ }^{\circ}$ 173-CEP/HUPE-CAAE; 0020.0.228.000-07. O estudo está também cadastrado na Comissão Nacional de Ética em Pesquisa com o $n^{\circ} 127.374$.

\section{RESULTADOS}

O grupo do estudo constituiu-se de 140 crianças, sendo 106 obesas ( 37 meninas e 69 meninos) e $34 \mathrm{com}$ sobrepeso (19 meninas e 15 meninos), com média de idade de 6,5 $\pm 2,3$ anos e média de escore $Z$ de IMC de $2,1 \pm 0,7$. As médias, os valores mínimos e máximos e os desvios-padrão das principais características clínicas e metabólicas das crianças estudadas estão na Tabela 1.

Do total de crianças avaliadas, $86(61,4 \%)$ tinham circunferência da cintura aumentada, seis $(4,3 \%)$ apresentaram hipertensão arterial e $39(27,9 \%)$ tinham acantose nigricans. A glicemia de jejum alterada foi encontrada em quatro crianças $(2,9 \%)$, enquanto HDLcolesterol baixo estava presente em $78(55,7 \%)$ e triglicérides altos em 28 (20\%). Do grupo estudado, 23 $(16,4 \%)$ preencheram o critério estabelecido para síndrome metabólica.

A sensibilidade, a especificidade e o índice de Youden obtidos para cada um dos pontos de corte do índice HOMA-IR estudados, tomando como desfecho a presença de síndrome metabólica, juntamente com os correspondentes intervalos de confiança, estão descritos na Tabela 2.

Tabela 1. Características clínicas e metabólicas das crianças com obesidade e sobrepeso estudadas, com suas médias, valores mínimos e máximos e desvios-padrão.

\begin{tabular}{lcccc} 
Características clínicas e metabólicas & Médias & Valores mínimos & Valores máximos & Desvios-padrão \\
Idade (anos) & 6,5 & 2 & 11 & 2,3 \\
\hline Escore Z de IMC & 2,1 & 1,0 & 4,7 & 0,7 \\
Glicose (mg/dL) & 85,9 & 64 & 115 & 8,3 \\
HDL-colesterol (mg/dL) & 44,7 & 20 & 95 & 11,4 \\
Triglicérides (mg/dL) & 91,8 & 23 & 229 & 41 \\
Insulina $(\mu \mathrm{IU} / \mathrm{mL})$ & 8,5 & 0,9 & 26,4 & 5,7 \\
HOMA-IR & 1,8 & 0,2 & 6,5 & 1,3 \\
\hline
\end{tabular}

IMC = índice de massa corporal; HOMA-IR = homeostasis model assessment for insulin resistance.

Tabela 2. Sensibilidade, especificidade e índice de Youden (com seus correspondentes intervalos de confiança) obtidos para cada um dos pontos de corte do índice HOMA-IR estudados, tomando como desfecho a presença de síndrome metabólica.

\begin{tabular}{lccccc} 
Pontos de corte de HOMA-IR & $\mathbf{2}$ & $\mathbf{2 , 5}$ & $\mathbf{3 , 1 6}$ & $\mathbf{3 , 4 5}$ & $\mathbf{3 , 8}$ \\
\hline Sensibilidade & 65,22 & 60,87 & 43,48 & 15 & 21,74 \\
& $(43,58-86,86)$ & $(38,75-82,99)$ & $(21,04-65,91)$ & $(0,00-33,15)$ & $(2,71-4,77)$ \\
Especificidade & 73,50 & 83,76 & 88,89 & 91,45 & 93,16 \\
& $(65,08-81,93)$ & $(76,65-90,87)$ & $(82,77-95,01)$ & $(85,96-96,95)$ & $(88,16-98,16)$ \\
Índice de Youden & 0,39 & 0,45 & 0,32 & 0,06 & 0,15 \\
& $(0,18-0,60)$ & $(0,24-0,66)$ & $(0,11-0,53)$ & $(-0,10-0,23)$ & $(-0,03-0,32)$ \\
\hline
\end{tabular}

HOMA-IR = homeostasis model assessment for insulin resistance. Fontes dos pontos de corte descritos: 2: Manios Y e cols. (22); 2,5: d'Annuzio G e cols. (25); 3, 16: Keskin M e cols. (17); 3,45: Cuartero BG e cols. (23); 3,8: Barja S e cols. (21). 
A curva ROC, construída a partir da representação gráfica dos valores de sensibilidade e especificidade encontrados, está representada na Figura 1. Dos pontos de corte de HOMA-IR testados, o que se situou no ombro da curva foi o de 2,5. Este valor também teve o maior índice de Youden.

A acurácia do teste, determinada pela área sob a curva ROC, foi de $72 \%$, com intervalo de confiança de 60 a 83, com nível de confiança de 95\%.

\section{DISCUSSÃo}

O grupo de crianças impúberes com obesidade e sobrepeso estudado foi grupo jovem, o que explica o fato de apenas $4,3 \%$ apresentar hipertensão arterial e 2,9\% glicemia de jejum alterada. Trata-se de grupo com obesidade não muito grave, cuja média de escore $\mathrm{Z}$ de IMC foi de 2,1. Sendo assim, o achado de alta prevalência de fatores de risco para doença cardiovascular aterosclerótica, como circunferência da cintura aumentada, HDLcolesterol baixo e triglicérides altos, é preocupante. A alta prevalência de acantose nigricans, considerada mar-

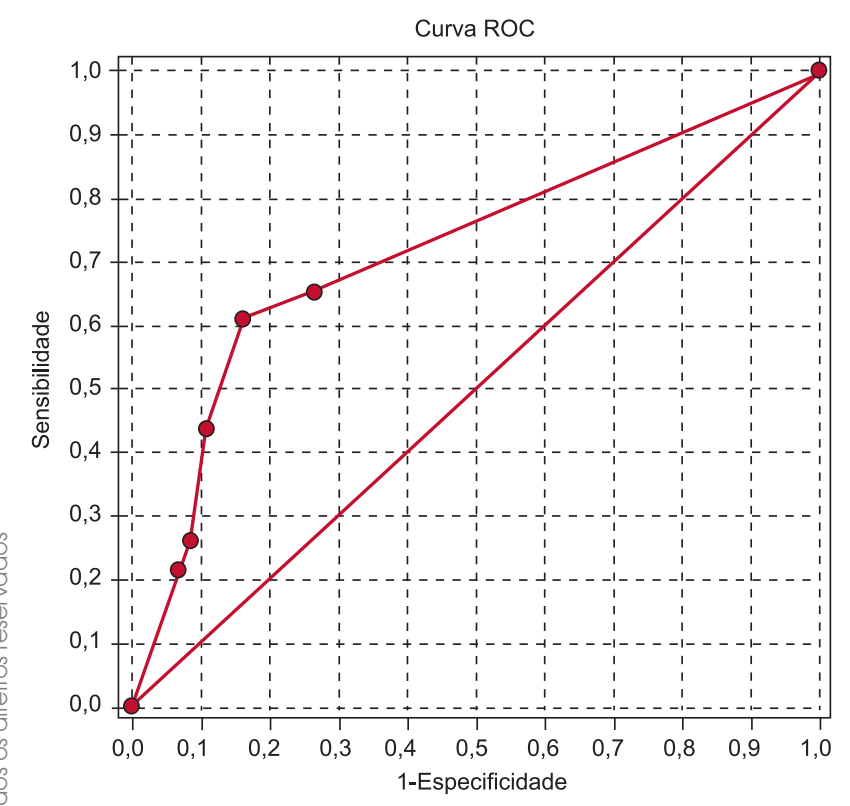

Os cinco pontos de corte representados no gráfico são, de baixo para cima: 3,$8 ; 3,45 ; 3,16 ; 2,5$ e 2 .

Figura 1. Curva ROC de pontos de corte de HOMA-IR para detecção de síndrome metabólica em crianças impúberes. cador de resistência insulínica, mesmo em crianças (3740), também chama a atenção.

A prevalência de síndrome metabólica, segundo o critério adaptado da IDF, também foi considerável no grupo estudado. Optou-se pela adaptação desta classificação por ser a única existente para crianças. Esta classificação, no entanto, só deve ser aplicada na clínica a crianças acima de 10 anos, por falta de evidências científicas referentes aos mais jovens (27).

Apesar de a definição desta síndrome ainda não ser consensual na infância, e ela não se tratar de uma doença, e sim de um agregado de fatores de risco para doença cardiovascular, a presença na criança obesa dos elementos que a compõem oferece subsídios clínicos para a existência, já nos impúberes, da resistência insulínica (11). Sendo assim, métodos para a detecção da resistência insulínica nesta população de risco são essenciais não só para seu diagnóstico, mas também para indicar sua gravidade e os fatores associados.

Um desses métodos é o índice HOMA-IR que, além de ser instrumento já validado para crianças, é de simples realização. Por ser mais factível que aqueles calculados a partir de campes, ou mesmo por TOTG, é mais aplicável a crianças menores, principalmente em estudos epidemiológicos.

O presente estudo determinou, a partir dos pontos de corte do índice HOMA-IR descritos na literatura para crianças e adolescentes, o valor de 2,5 como aquele que mais bem identificou síndrome metabólica em crianças impúberes obesas e com sobrepeso. A sensibilidade para este ponto de corte foi de 61\%, o que significa que, ao utilizá-lo em crianças obesas e com sobrepeso impúberes, $61 \%$ delas estará sendo corretamente diagnosticada como tendo síndrome metabólica, enquanto a especificidade foi de $84 \%$, o que vale dizer que, ao utilizá-lo, $84 \%$ delas estará sendo corretamente diagnosticada como não tendo síndrome metabólica.

A particularidade do estudo realizado foi a faixa etária estudada, uma vez que são escassos os estudos sobre o tema em crianças em tenra idade.

A grande totalidade dos trabalhos inclui crianças já púberes, como o de Keskin e cols., que construíram a curva ROC utilizando como padrão-ouro a presença de resistência insulínica, com base na soma dos níveis de insulina após TOTG. Estes autores chegaram ao ponto de corte de HOMA-IR de 3,16, com sensibilidade de $76 \%$ e especificidade de 66\% (17). Atabek e Pirgon (18) realizaram estudo semelhante e chegaram ao ponto de 
corte de 2,7 , com sensibilidade de $80 \%$ e especificidade de $59 \%$.

Tresaco e cols. (24) também incluíram crianças púberes em seu estudo, e construíram a curva ROC utilizando como padrão-ouro a presença de síndrome metabólica, propondo ponto de corte de HOMA-IR próximo de 3 , com sensibilidade de $81 \%$ e especificidade de $76 \%$.

Dos estudos que elegeram os pontos de corte de HOMA-IR fundamentados em medidas de dispersão populacional, apenas o estudo multicêntrico italiano de d'Annunzio e cols. (25) forneceu dados sobre impúberes em separado, chegando ao ponto de corte de HOMA-IR de 2,5 ao utilizar o percentil 95.

Diante das suas peculiaridades, as crianças impúberes devem ser estudadas em separado daquelas já em puberdade, na qual os efeitos dos esteróides hormonais já se fazem presentes. A este respeito, sabe-se que a insulina $(41,42)$ e o índice HOMA-IR $(39)$, bem como a frequiência de síndrome de resistência insulínica $(43)$ aumentam com a evolução da puberdade.

Embora os estudos exclusivos em impúberes sejam escassos, alguns fatores de risco para doença cardiovascular já estão presentes, conforme pode-se observar no presente trabalho, o que torna o grupo de crianças obesas impúberes um grupo candidato ao estudo da fase inicial da resistência insulínica, a despeito das dificuldades técnicas inerentes à tenra idade.

Recentemente, já se têm demonstrado, por meio de estudos longitudinais, que crianças com características da síndrome metabólica se tornam adultos com síndrome metabólica (44-46), que, sabidamente, implica risco para doença cardiovascular aterosclerótica. Busca-se agora, mais do que nunca, definição consensual da síndrome na criança (26), e, para tal, é importante a definição de seus componentes, assim como de seus pontos de corte, a fim de mais bem identificar aquelas em maior risco cardiovascular. As crianças obesas com resistência insulínica compõem o grupo para o qual a alocação de recursos e pesquisas poderia ser direcionada.

O ponto de corte de HOMA-IR encontrado no grupo estudado é aplicável apenas a crianças obesas e com sobrepeso de serviços ambulatoriais de pediatria geral. Em razão do aumento da prevalência da obesidade infantil, implicando aumento de morbimortalidade por doença cardiovascular na idade adulta, o estabelecimento de um ponto de corte para HOMA-IR pode ser bastante útil na identificação das crianças em maior risco cardiovascular.

As limitações do presente estudo foram, principalmente, aquelas impostas pela idade tenra das crianças estudadas, para a qual não há consenso em relação à definição de síndrome metabólica. Nesta faixa etária existem também as dificuldades técnicas para a realização de procedimentos mais apurados para a definição da resistência insulínica, como aqueles realizados após TOTG e os clampes de insulina e glicose.

Os autores recomendam cautela ao se identificar crianças em idade tenra supostamente em maior risco para doenças cardiovasculares, uma vez que os estudos para a faixa etária são escassos. A abordagem destas crianças deve manter seu foco na profilaxia, ou seja, na promoção de estilo de vida saudável.

Agradecimentos: Os autores agradecem aos colegas do Laboratório Central do HUPE-UERJ, especialmente ao Dr. Vagner Ismelim Lobão, pela realização das análises bioquímicas. A pesquisa faz parte do projeto de tese de doutoramento da autora principal Isabel Rey Madeira, sob orientação da co-autora Maria Alice Neves Bordallo. O projeto recebeu Auxílio à Pesquisa (APQ1) da Fundação Carlos Chagas Filho de Amparo à Pesquisa do Estado do Rio de Janeiro (Faperj), Protocolo $n^{\circ}$ 170491, de 2007. Os autores declaram não haver conflito de interesse que possa interferir na imparcialidade do trabalho científico.

\section{REFERÊNCIAS}

1. Styne DM. Childhood and adolescent obesity. Prevalence and significance. Pediatr Clin North Am. 2001;48:823-54.

2. IBGE. Pesquisa de orçamentos familiares - POF 2002-2003 [acessado em: 2008 Mar 7]. Disponível em: http://www.ibge. gov.br/home/presidencia/noticias/noticia_impressao. php?idnoticia=278.10-10-2005.

3. Rosamond WD, Chambless LE, Folsom AR, Aaron R, Cooper $L S$, Cornwill DE, et al. Trends in the incidence of miocardial infarction and in mortality due to coronary heart disease, 1987 to 1994. N Engl J Med. 1998;339:861-7.

4. Berenson GS, Srinivasan SR, Bao W, Newman WP, Tracy RE, Wattigney WA. Association between multiple cardiovascular risk factors and atherosclerosis in children and young adults. The Bogalusa Heart Study. N Engl J Med. 1998;338:1650-6.

5. Reaven GM. Role of insulin resistance in human disease. Diabetes. 1988;37:1595-607.

6. Barja S, Arteaga A, Acosta AM, Hogson, MI. Insulin resistanse and other expressions of metabolic syndrome in obese Chilean children. Rev Med Chil. 2003;131:259-68.

7. Invitti C, Guzzaloni G, Gilardini L, Morabito F, Viberti G. Prevalence and concomitants of glucose intolerance in European obese children and adolescents. Diabetes Care. 2003;26:118-24. 
8. Steinberger J, Daniels SR. Obesity, insulin resistance, diabetes and cardiovascular risk in children. Circulation. 2003; 107:1448-53.

9. Grundy SM. Obesity, metabolic syndrome and cardiovascular disease. J Clin Endocrinol Metab. 2004;89:2595-600.

10. Papadopoulou-Alataki E, Papadopoulou-Legbelou K, Doukas L, Karatzidou K, Pavlitou-Tsiontsi A, Pagkalos E. Clinical and biochemical manifestations of syndrome $X$ in obese children. Eur J Pediatr. 2004;163:573-9.

11. Ten S, MacLaren N. Insulin resistance syndrome in children. J Clin Endocrinol Metab. 2004;89:2526-39.

12. Matthews DR, Hosker JP, Rudenski AS, Naylor BA, Treacher DF, Turner RC. Homeostasis model assessment: insulin resistance and $ß$-cell function from fasting plasma glucose and insulin concentrations in man. Diabetologia. 1985;28:412-9.

13. Vasques ACJ, Rosaldo LEFPL, Alfenas RCG, Geloneze B. Análise crítica do uso dos índices do homeostasis model assessment (HOMA) na avaliação da resistência à insulina e capacidade funcional das células-ß pancreáticas. Arq Bras Endocrinol Metab. 2008;52;32-9.

14. Guzzaloni G, Grugni G, Mazzilli G, Moro D, Morabito F. Comparison between beta-cell function and insulin resistance indexes in pre-pubertal and pubertal obese children. Metabolism. 2002;51:1011-6.

15. Conwell LS, Trost SG, Brown, WJ, Batch JA. Indexes of insulin resistance and secretion in obese children and adolescents. $A$ validation study. Diabetes Care. 2004;27:314-9.

16. Hoffman RP, Vicini P, Cobelli C. Pubertal changes in HOMA and Quicki: a relationship to hepatic and peripheral insulin sensitivity. Pediatr Diabetes. 2004;5:122-5.

17. Keskin M, Kurtoglu S, Kendirci M, Atabek ME, Yazuci C. Homeostasis model assessment is more reliable than fasting glucose/insulin ratio and quantitative insulin sensitivity check index for assessing insulin resistance among obese children and adolescents. Pediatrics. 2005;115:500-3.

18. Atabek ME, Pirgon O. Assessment of insulin sensitivity from measurements in fasting state and during an oral glucose tolerance test in obese children. J Pediatr Endocrinol Metab. 2007;25:187-95.

19. Uaifo GI, Fallon EM, Chin J, Elberg J, Parikh SJ, Yanovski JA. Indices of insulin action, disposal, and secretion derived from fasting samples and clamps in normal glucose-tolerant black and white children. Diabetes Care. 2002;25:2081-7.

20. Gungor, N, Saad R, Janosky J, Arslanian S. Validation of surrogate estimates of insulin sensitivity and insulin secretion in children and adolescents. J Pediatr. 2004;144:47-55.

21. Barja S, Arteaga A, Acosta AM, Hogson, MI. Resistencia insulínica y outras expressiones del síndrome metabólico em niños obesos chilenos. Rev Med Chil. 2003;131:259-68.

22. Manios $Y$, Moschonis G, Kourlaba G, Bouloubasi Z, Grammatikaki E, Spyridaki A, et al. Metabolism prevalence and independent predictors of insulin resistance in children from Crete, Greece: the children study. Diabet Med. 2007;25:65-72.

23. Cuartero BG, Lacalle CG, Lobo CJ, Vergaz AG, Rey CC, Villar MJA, et al. Índice HOMA y Quicki, insulina y peptide $\mathrm{C}$ en niños sanos. Puntos de corte de riesgo cardiovascular. An Pediatr (Barc). 2007;66:481-9.

24. Tresaco B, Bueno G, Pineda I, Moreno LA, Garagorri JM, Bueno M. Homeostatic model assessment (HOMA) index cut-off values to identify the metabolic syndrome in children. J Physiol Biochem. 2005;61:381-8.
25. d'Annunzio G, Vanelli M, Meschi F, Pistorio A, Caso M, Pongigline $\mathrm{C}$, et al. The SIDEP Study Group. Valori normali di HOMA-IR in bambini e adolescenti: studio multicentrico italaiano. Quad Pediatr. 2004;3:44.

26. Ford ES, Li C. Defining the metabolic syndrome in children and adolescents: will the real definition please stand up? J Pediatr. 2008;152:160-4.

27. Zimmet $P$, Alberti G, Kaufman F, Tajima N, Silink M, Arslanian $\mathrm{S}$, et al. The metabolic syndrome in children and adolescents. Lancet. 2007;369:2059-61.

28. Marchall WA, Tanner JM. Variations in the pattern of pubertal changes in girls and boys. Arch Dis Child. 1969;44:291-303.

29. Abrantes MM, Lamournier JA, Colosimo EA. Prevalência de sobrepeso e obesidade em crianças e adolescentes das regiões sudeste e nordeste. J Pediatr (Rio J). 2002;78:327-34.

30. Fleiss JL, Levin BA, Levin B, Paik MC. Statistical methods for rates and proportions. 3 ${ }^{a}$ ed. Oxford: Wiley-InterScience; 2003.

31. Fernández JR, Redden DT, Pietrobelli A, Allison DB. Waist circumference percentiles in nationally representative samples of African-American, European-American, and Mexican-American children and adolescents. J Pediatr. 2004;145:439-44.

32. Hoekelman RA. Exame físico de lactentes e crianças - pressão arterial. In: Bickley LS, Hoekelman RA, editors. Bates propedêutica médica. 7. ed. Rio de Janeiro: Guanabara Koogan; 1999. p. 617-20.

33. Rosner B, Prineas R, Loggie J, Daniels SR. Percentiles for body mass index in US children 5 to 17 years of age. J Pediatr. 1998;132:211-22.

34. National High Blood Pressure Education Program Working Group on High Blood Pressure in Children and Adolescents. The fourth report on the diagnosis, evaluation, and treatment of high blood pressure in children and adolescents. Pediatric. 2004;114:555-76.

35. Giuliano ICB, Caramelli B, Pellanda L, Duncan B, Mattos S, Fonseca FH. Sociedade Brasileira de Cardiologia. I Diretriz de Prevenção da Aterosclerose na Infância e na Adolescência. Arq Bras Cardiol. 2005;85 Suppl VI:1-36.

36. Fletcher RH, Fletcher SW. Diagnóstico - curva ROC. In: Fletcher RH, Fletcher SW, editores. Epidemiologia Clínica - Elementos Essenciais. 4. ed. Porto Alegre: Artmed; 2006. p. 63-4.

37. Yamazaki $\mathrm{H}$, Ito $\mathrm{S}$, Yoshida $\mathrm{H}$. Acanthosis nigricans is a reliable cutaneous marker of insulin resistance in obese Japanese children. Pediatr Int. 2003;45:701-5.

38. Urrutia-Rojas X, Menchaca J, Wadley W, Ahmad N, Lacko A, Bae $S$, et al. Cardiovascular risk factors in Mexican-American children at risk for type 2 diabetes mellitus. J Adolesc Health. 2004;34:290-9.

39. Copeland K, Pankratz K, Cathey V, Immohotichey P, Maddox J, Felton $B$, et al. Acanthosis nigricans, insulin resistance (HOMA) and dyslipidemia among native American children. J Okla State Med Assoc. 2006;99:19-24.

40. Miura N, Ikezaki A, Iwama S, Matsuoka H, Ito K, Sugihara S. Genetic factors and clinical significance of acanthosis nigricans in obese japanese children and adolescents. Acta Paediatr. 2006;95:170-5.

41. Aycan Z, Berberoglu M, Ocal G, Evliyaoglu O, Adiyaman P, Deda $G$, et al. Relationship between plasma leptin, insulin and tumor necrosis factor alpha in obese children. J Pediatr Endocrinol Metab. 2005;18:275-84.

42. Burrows AR, Leiva BL, Burgueño AM, Maggi MA, Giadrosic $R V$, Díaz BE, et al. Sensibilidad insulínica en niños de 6 a 15 
años: associación con estado nutricional y pubertad. Rev Med Chil. 2006;134:1417-26.

43. Druet C, Dabbas B, Baltakse V, Payen C, Jouret B, Baud C, et al. Insulin resistance and the metabolic syndrome in obese French children. Clin Endocrinol (Oxf). 2006;64:672-8.

44. Shaibi GQ, Goran MI. Examining metabolic syndrome definitions in overweight Hispanic youth: a focus on insulin resistance. J Pediatr. 2008;152:171-6.

45. Sun SS, Liang R, Huang TTK, Daniels SR, Arslanian S, Liu K, et al. Childhood obesity predicts adult metabolic syndrome: the fels longitudinal study. J Pediatr. 2008;152:191-200.
46. Morrison JA, Friedman LA, Wang P, Gluec CJ. Metabolic syndrome in childhood predicts adult metabolic syndrome and type 2 diabetes mellitus 25 to 30 years later. J Pediatr. 2008; 152:201-6.

Endereço para correspondência:

Isabel Rey Madeira

Rua João da Mata, 128, Tijuca

2051 1-260 Rio de Janeiro RJ

E-mail: isamadeira@oi.com.br 